\title{
Multifocal Myoclonus after Stroke and Rehabilitation - A Case Report
}

\author{
Kavitha Andiappan* and Lydia Abdul Latif \\ Department of Rehabilitation Medicine, University Malaya Medical Centre, Malaysia
}

Corresponding author: Kavitha Andiappan, Department of Rehabilitation Medicine, Medical Faculty, $12^{\text {th }}$ floor, Menara Selatan, University Malaya Medical Centre, 59100, Kuala Lumpur, Malaysia, Tel: 60123897001; E-mail: kavitha_andiappan@yahoo.com

Received: Jun 03, 2017; Accepted: Jun 23, 2017; Published: Jun 27, 2017

Citation: Andiappan K, Abdul Latif L (2017) Multifocal Myoclonus after Stroke and Rehabilitation - A Case Report. J Neurol Neurosci 8:3.

\section{Abstract}

Movement disorders are a rare recognized complication of stroke. Different types of hyperkinetic and hypokinetic movement disorders have been reported and can be seen after ischemic and haemorrhagic strokes. Failure to address these involuntary movements poses a challenge for post-stroke intensive multidisciplinary rehabilitation. We describe a patient presenting with multifocal myoclonus after an Anterior Cerebral Artery (ACA) territory infarct whose symptoms were brought under control with clonazepam. Additionally, we discussed on our experience and the challenges faced in the diagnostic process, management, and rehabilitation of this patient.

Keywords: Myoclonus after stroke; Rehabilitation

\section{Introduction}

Movement disorders are a recognised complication of stroke. Reported frequency of involuntary movements following stroke ranges between 1-4\% [1-3]. Although rare, many different varieties of abnormal movement can be found after a stroke either acutely or as a delayed sequel. They can be hyperkinetic (most commonly hemichorea-hemiballismus) or hypokinetic (most commonly vascular parkinsonism). Most are caused by lesions in the basal ganglia or thalamus but can occur with strokes at many different locations in the motor circuit [3]. Among these, myoclonus is one of the rarest form of movement disorder in patients with stroke [1]. Myoclonus is described as sudden, brief, shock like, involuntary movements caused by muscular contractions [4]. Myoclonus after a stroke has been so far reported to be focal or segmental with posterior circulation strokes, particularly midbrain, pontine, and thalamic strokes $[1,2]$. However, no multifocal myoclonus following a stroke has been reported prior to this [1].

\section{Case Report}

A 59-year-old gentleman of no known medical illness initially presented to the hospital with one day history of sudden onset of left-sided limb weakness. The blood pressure upon presentation was $190 / 107 \mathrm{mmHg}$. 2 days prior to the presentation, he had what he described as a sudden twitching of the left lower limb lasting for about 5 minutes and resolved spontaneously. He was conscious and aware of the involuntary movement but was unable to control it. Upon presentation to the hospital, it was noted that he had left upper motor neuron facial nerve palsy with left-sided hemiparesis with a general muscle power of 4/5 according to the Medical Research Council (MRC) grading system on the left side. Glasgow Coma Scale (GCS) level was full at all times. Computer Tomography (CT) scan of the brain showed less well defined hypodensity at the right centrum semiovale.

On the 2nd day, the left-sided weakness progressively worsened till it was dense flaccid left hemiparesis. There were also increasing occurrences of jerky movements of the left upper limb, abdomen, and lower limb with each episode lasting 2 to 3 minutes each time without any loss of consciousness. Repeated CT scan of the brain showed early subacute right ACA territory infarct (Figure 1). Electroencephalogram (EEG) did not reveal any epileptic form discharges throughout the recording despite the fact that the patient had 1 episode of involuntary movement of the abdomen during the EEG procedure. He was diagnosed to have right ACA infarct with epilepsia partialis continua (EPC). Antiepileptic agent, oral sodium valproate $400 \mathrm{mg}$ thrice a day, was initiated. The involuntary movements became less frequent with medication.

On the 10th day of admission, the patient was transferred to rehabilitation care to undergo active multidisciplinary rehabilitation. He was severely dependant on all aspects of personal activity of daily living at the point of transfer. Upon transfer, his involuntary movement worsened without any clouding of consciousness. They were described as jerky movements of the left upper limb lasting 2-3 minutes, each time occurring once every hour at average and resolving spontaneously. Over the next few days, the involuntary jerky movements progressively involved the right upper limb as well. A second antiepileptic agent, oral carbamazepine $200 \mathrm{mg}$ thrice a day, was added to the prescription. The addition of the antiepileptic medication managed to reduce the frequency of involuntary movement, but not significantly.

Intensive rehabilitation was unable to commence as the involuntary movement affected the patient physically, psychologically, and emotionally. This condition caused deep frustration to him as it disturbed him in many ways including 
poor sleep. The psychological and emotional state of the patient caused low motivation and severe inattention during therapy sessions. His Patient Health Questionnaire-9 (PHQ-9) score was 14 indicating moderate depression for which he was started on sertraline, a Selective Serotonin Reuptake Inhibitor (SSRI). Furthermore, the therapists were not very comfortable in conducting therapy sessions at the gym in view of the given diagnosis of epilepsy and the frequent attacks. During this time comprehensive slow-stream rehabilitation approach was initiated focusing on general reconditioning to prevent deconditioning and further complications of immobilization which included nutrition optimization, proper bladder, bowel and skin care, cardiopulmonary training to improve cardiopulmonary function, proper positioning and joint protection strategies, attaining head and truncal control, hemiplegic shoulder joint protection and limb mobilization including stretching exercises to prevent contracture.

On the $23^{\text {rd }}$ day post stroke a repeat EEG revealed normal findings. The diagnosis was revised to multifocal myoclonus after stroke. The presentation of the patient was not in line with the clinical picture of EPC. EPC is characterized by focal motor clonic activity restricted to one body part recurring at fairly regular intervals lasting for a minimum of 1 hour. The EPC typically has unilateral involvement with evident post ictal weakness $[5,6]$. Patient's EEG both times were normal and not suggestive of EPC.

Once the diagnosis was revised, oral clonazepam of $0.5 \mathrm{mg}$ twice a day was initiated while simultaneously tapering off the antiepileptic medications. The involuntary movement of the upper limbs reduced drastically occurring very rarely (at an average of twice to thrice a week as reported by the patient). The patient was able to be subjected to intensive inpatient multidisciplinary rehabilitation once the myoclonic jerks were under control. He became more cheerful and motivated. His repeated PHQ- 9 score was 0 and the antidepressant, sertraline, was stopped. He progressed steadily thereafter during his rehabilitative course showing tremendous neurological and functional recovery. His Modified Barthel Index (MBI) score improved from 37 prior to rehabilitation to 52 indicating moderate level of dependency on discharge. His Trunk Control Test (TCT) also improved from 24 to 74 upon discharge suggesting improved truncal balance.

The patient was discharged home after 2 months of multidisciplinary rehabilitation. His progress and rehabilitation is continued and monitored as part of outpatient care, focusing on community integration.

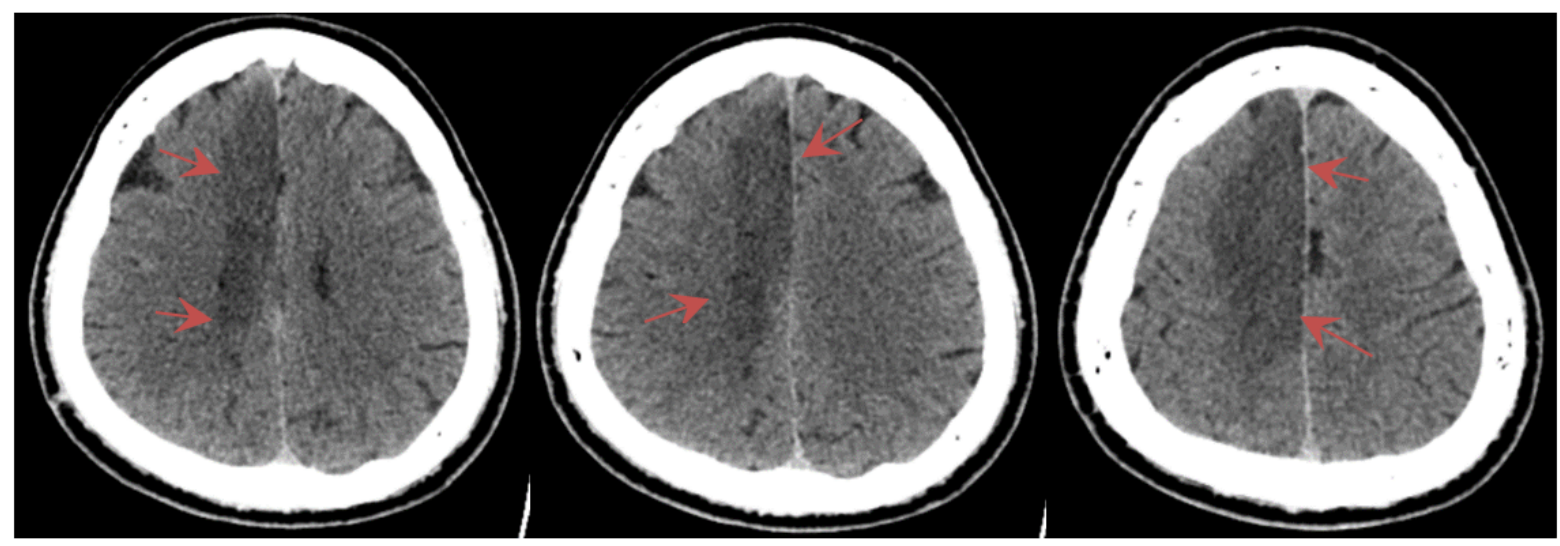

Figure 1 The arrows showed fairly well defined hypodense at the right ACA territory in CT brain.

\section{Discussion}

The frequency of post-stroke abnormal movements has been estimated to vary between $1 \%$ and $4 \%$ of all strokes [1-3]. In a study based on the hospital stroke registry in Ecuador, South America, 3.7\% of 1500 stroke patients developed post stroke movement disorder [2]. A review of the hospital-based Lausanne Stroke Registry identified a prevalence of $1 \%$ and an estimated incidence of $0.08 \%$ per year [7]. These involuntary movement after stroke affects both sexes equally and the mean age at onset is typically in the sixth and seventh decades of life, but can range from early childhood to age 90 years [2,7]. Although a number of different locations within the brain have been identified to be responsible for abnormal movements when affected by stroke, the basal ganglia are most often implicated in movement disorders following a stroke. The basal ganglia circuitry involves direct and indirect pathways which act as a cortical feedback loop in which signals from the neo-cortex are relayed through the striatum, pallidum, and thalamus back to the cortex to control voluntary motor movements. Interruption of direct or indirect pathways by focal lesions may lead to movement disorders. One alternate theory suggests that the dysfunction of dopaminergic and gamma-Aminobutyric acid (GABA) ergic systems as a result of neuronal injury may lead to after stroke movement disorders. In particular, GABA mediates the majority of fast inhibitory synaptic transmission in the central nervous system (CNS) and the alteration in the inhibitory mechanisms may explain the neuronal hyperexcitability that underlies some forms of myoclonus [8].

Myoclonus is one the form of hyperkinetic movement disorder after stroke that has been rarely reported [3]. Myoclonus is a positive phenomenon described as a sudden, 
involuntary, brief, irregular, jerk-like synchronized muscle contractions in single or multiple muscle groups. So far only focal or segmental myoclonus has been reported in posterior circulation stroke particularly with lesions in midbrain, pontine, and thalamus [1,2]. This patient presented with multifocal myoclonus occurring immediately following a right ACA territory stroke which had not been reported before [1]. The presence of multifocal involuntary movement following unilateral brain infarct may be explained by the recent advances in functional brain imaging which suggests that although the histopathological hallmark of movement disorders is located in the basal ganglia, they can be caused by alterations within functional whole brain networks rather than localized neurodegeneration [9].

The treatment of myoclonus aims to enhance the deficient GABAergic inhibitory neurotransmission. The two most commonly used GABAergic drugs are clonazepam and sodium valproate. Other tried treatments include levetiracetam, piracetam, primidone and acetazolamide. Treatment should begin with a single agent although eventually several drugs in combination may be required. Clonazepam is effective in all types of myoclonus. Side-effects include sedation, vertigo, behavioural changes and tolerance and it is contraindicated in people with acute narrow-angle glaucoma and liver problems [10]. Sodium valproate is effective in cortical and subcortical myoclonus. Side-effects include drowsiness, weight gain, tremor, nausea and alopecia. It is contraindicated in hepatic insufficiency [10]. Piracetam is less sedating and may be as effective as levetiracetam but both drugs should be used with caution in renal impairment [11]. Primidone can be tried but it causes drowsiness, confusion and falls. Acetazolamide is a carbonic anhydrase inhibitor that has been reported to be useful in the treatment of myoclonus because it is able to enhance the GABA effect and the GABA levels in brain however, acetazolamide necessitates electrolyte monitoring.

The timely diagnosis of myoclonus following a stroke is essential to facilitate intensive multidisciplinary rehabilitation. It is proven that early and intensive multidisciplinary rehabilitation in acute phase of stroke leads to improved functional outcomes. Myoclonus after stroke is a hindering factor in initiation of intensive rehabilitation as it affects the physical, psychological and emotional state of a person as seen in our patient. Frequent myoclonic jerks affect therapeutic exercises which promotes motor recovery. It also interferes with the sleep hygiene which in turn affects the mood stability. In the process of identifying and managing the involuntary movements accordingly, our patient lost nearly 2 weeks of active rehabilitation. Therefore, the diagnosis of movement disorder after stroke should always be considered in the event of involuntary movement following a stroke and treated accordingly in a swift manner. This would facilitate early initiation of multidisciplinary rehabilitation leading to meaningful improvement in terms of motor recovery and functional outcomes.

\section{Conclusion}

Although rare, many different varieties of abnormal movement can be found after a stroke. Presentation of these involuntary movements may differ from case to case and it is shown to delay the post-stroke active rehabilitation process. Timely diagnosis and prompt intervention of movement disorder after stroke is essential to initiate an early intensive multidisciplinary rehabilitation programme facilitating motor recovery and favourable functional outcomes.

\section{References}

1. Ghika J, Bogousslavsky J (1995) Abnormal movements. In: Bogousslavsky J, Caplan L, (eds) Stroke syndrome. Cambridge University Press, NY, USA 91-101.

2. Alarcon F, Zijlmans JC, Duenas G, Cevallos N (2004) Post-stroke movement disorders: A report of 56 patients. J Neurol Neurosurg Psychiatry 75: 1568-1574.

3. Handley A, Medcalf P, Hellier K, Dutta D (2009) Movement disorders after stroke. Age Ageing 38: 260-266.

4. Marsden CD, Obese JA, Zarranz JJ, Lang AE (1985) The anatomical basis of symptomatic hemidystonia. Brain 108: 463-483.

5. Gastaut H (1968) Semeiology of myoclonus and analytic nosology of myoclonic syndromes. Rev Neurol 119: 1-30.

6. Bancaud J, Bonis A, Trottier S, Talairach J, Dulac O (1982) Continuous partial epilepsy: Syndrome and disease. Rev Neurol 138: 803-814.

7. Ghika SF, Ghika J, Regli F, Bogousslavsky J (1997) Hyperkinetic movement disorders during and after acute stroke: The Lausanne Stroke Registry. J Neurol Sci 146: 109-116.

8. Krauss GL, Mathews GC (2003) Similarities in mechanisms and treatments for epileptic and nonepileptic myoclonus. Epilepsy Curr 3: 19-21.

9. Holtbernd F, Eidelberg D (2012) Functional brain networks in movement disorders. Curr Opin Neurol 25: 392-401.

10. Sawle G (1999) Movement disorders in clinical practice. Martin Dunitz, Oxford.

11. Ikeda A, Shibasaki H, Tashiro K, Mizuno Y, Kimura J (1996) Clinical trial of piracetam in patients with myoclonus, nationwide multiinstitution study in Japan. The Myoclonus/Piracetam Study Group. Mov Disord 11: 691-700. 